\title{
Suicídios nos Municípios Brasileiros: uma Abordagem Espacial para Dados Censurados
}

\section{Suicides in the Brazilian Municipalities: a Spatial Approach for Censored Data}

\author{
Samia Mercado Alvarenga ${ }^{\mathrm{a}}$ \\ Marco Antônio Jorge ${ }^{\mathrm{b}}$ \\ Pedro Vasconcelos Maia do Amaral ${ }^{\mathrm{a}}$
}

\begin{abstract}
Resumo: O suicídio é reconhecido internacionalmente como um problema de saúde pública que figura entre as dez causas de mortes mais frequentes entre a população em geral, superando a quantidade de mortes por conflitos armados. No Brasil, segundo dados do Sistema de Informações de Mortalidade, para cada suicídio registrado, estima-se que existem mais de 20 tentativas não reportadas. $\mathrm{O}$ sub-registro, aliado à raridade e às características inerentes a sua distribuição, torna o fenômeno ainda mais complexo, especialmente no contexto brasileiro, em que as dimensões continentais comportam heterogeneidades socioeconômicas e culturais. No intuito de verificar a influência de fatores socioeconômicos na presença de interações espaciais, utiliza-se um modelo tobit espacialmente defasado aplicado a dados municipais de 2010. A abordagem empírica adotada representa um importante avanço na literatura, já que, além de considerar a autocorrelação espacial a um nível de agregação que, até então, não era comum às pesquisas a respeito do suicídio, também oferece um tratamento para variáveis censuradas. Os principais resultados corroboram estudos anteriores acerca da influência significativa dos fatores socioeconômicos e confirmam efeitos multiplicadores espaciais, consistentes com os postulados durkheimianos sobre o efeito-contágio. Portanto, é desejável que políticas públicas para contenção e prevenção do suicídio levem em consideração não só aspectos socioeconômicos e demográficos, como também a distribuição territorial das ocorrências e a interação espacial existente entre elas.
\end{abstract}

Palavras-chave: suicídio; econometria espacial; tobit; municípios brasileiros.

Abstract: Suicide is recognized internationally as a public health problem that ranks among the ten most frequent causes of death among the general population, surpassing the number of deaths from armed conflict. In Brazil, according to data from the Mortality Information System, for each registered suicide, it is estimated that there are over

a Universidade Federal de Minas Gerais (UFMG), Centro de Desenvolvimento e Planejamento Regional (CEDEPLAR), Doutorado em Economia. Belo Horizonte, Minas Gerais, Brasil.

b Universidade Federal de Sergipe (UFS), Departamento de Economia (DEE). São Cristóvão, Sergipe, Brasil. 
twenty attempts not reported. Under-registration, coupled with rarity and the inherent characteristics of its distribution, makes the phenomenon even more complex, especially in the Brazilian context, where the continental dimensions carry socioeconomic and cultural heterogeneities. In order to verify the influence of socioeconomic factors in the presence of spatial interactions, a spatially distributed Tobit model is applied to municipal data of 2010. The empirical approach adopted represents an important advance in the literature, since, besides considering the spatial autocorrelation a level of aggregation that hitherto was not common to those surveyed regarding suicide, also offers a treatment for censored variables. The main results corroborate previous studies about the significant influence of socioeconomic factors and confirm spatial multiplier effects, consistent with the Durkheimian postulates about the contagious effect. Therefore, it is desirable that public policies to contain and prevent suicide take into account not only socioeconomic and demographic aspects, but also the territorial distribution of occurrences and the spatial interaction between them.

Keywords: suicide; spatial econometrics; tobit; Brazilian municipalities.

JEL Classification: I12; C21; C24.

\section{1 lntrodução}

O suicídio é um fenômeno complexo em que o indivíduo põe fim de forma intencional à própria vida. De natureza eminentemente humana, tal ato esteve sempre presente ao longo da história e em todos os espectros culturais. Entretanto, o aumento das ocorrências, nos últimos 50 anos, tem despertado o interesse da comunidade científica em torno das suas motivações. Segundo a Organização Mundial da Saúde (WORLD HEALTH ORGANIZATION, 2014), nesse período as mortes por suicídio cresceram mais de $60 \%$ em todo o globo, superando o total de mortes em conflitos armados.

Em números absolutos, as mortes por suicídio são maiores nos países de baixa e de média renda, os quais concentram o maior contingente populacional do mundo, respondendo por cerca de 75\% das ocorrências globais de suicídio (WORLD HEALTH ORGANIZATION, 2016). Porém, os estudos voltados a essa temática não acompanharam de forma proporcional o número dos casos, concentrando-se majoritariamente em nações desenvolvidas (SETTI, 2017). Em comum com tais investigações, sejam no âmbito internacional (YANG, 1992; JALLES; ANDERSEN, 2015), ou no nacional (SHIKIDA; ARAÚJO JÚNIOR; GAZZI, 2006; LOUREIRO; MOREIRA; SACHSIDA, 2013; DE FRAGA; MASSUQUETTI; GODOY, 2017), encontra-se a influência significativa de variáveis socioeconômicas.

Os resultados obtidos, em geral, corroboram o que já aponta a Organização Mundial da Saúde, a qual garante que as dificuldades econômicas e sociais figuram como um fator precipitante do suicídio (WORLD HEALTH ORGANIZATION, 
2016). Contudo, ainda não há consenso sobre a forma como esses fatores interagem com aspectos demográficos e, sobretudo, espaciais, permanecendo pouco explorado o papel das interações geográficas nas mortes por suicídio (AMARAL, 2018). Esse cenário é ainda mais complexo no Brasil devido à sua extensão territorial, que, aliada à diversidade étnico-cultural, favorece o predomínio da heterogeneidade no fenômeno (ALMEIDA et al., 2015).

Segundo dados do Sistema de Informações de Mortalidade, em média 11 mil brasileiros tiram a própria vida anualmente, o que corresponde a aproximadamente 26 ocorrências diárias. Para cada suicídio, estima-se a existência de pelo menos 20 tentativas não registradas (DATASUS, 2017). Além de sub-reportadas, as estatísticas do país guardam uma dissimilaridade regional importante, sendo menos intensas nos estados nordestinos e mais elevadas na região Sul, onde o quantitativo de mortes voluntárias é comparável e, por vezes, até superior aos altos índices de países escandinavos (BOTEGA, 2007). Esse panorama faz do Brasil um ambiente propício para se investigar os suicídios na presença de fatores econômicos de risco que coexistem com um contexto de diversidade cultural e étnica.

Com base no exposto, levanta-se como hipótese a existência de efeito multiplicador, de modo que a ocorrência de mortes voluntárias em determinada localidade pode estar relacionada às mortes voluntárias do seu entorno, o que vai ao encontro dos postulados de Durkheim (2005) acerca do contágio, embora ao nível individual. Diante disso, pergunta-se: qual a influência de fatores socioeconômicos nos suicídios considerando-se interações espaciais? Para responder ao problema de pesquisa, propõe-se a utilização de um modelo tobit espacialmente defasado aplicado aos municípios brasileiros no ano de 2010. O período escolhido para o corte transversal visa abarcar variáveis de controle, as quais são melhor disponibilizadas em anos censitários. Nesse sentido, o objetivo do presente trabalho é verificar a influência de fatores socioeconômicos na presença de interações espaciais.

Regressões espaciais para os suicídios no Brasil já foram utilizadas por Gonçalves, Gonçalves e Oliveira Júnior (2011), Siqueira (2017) e Amaral (2018), os quais trabalham com dados agregados por microrregião. Ao se analisar as taxas de suicídios ao nível de municípios, a forte incidência de zeros em função da baixa frequência do fenômeno requer tratamento específico devido à distribuição da variável. Nesse sentido, torna-se oportuno a utilização de modelos espaciais que tratem as estatísticas de suicídio como uma medida censurada em zero (ARAÚJO; GARCIA, 2019). Assim, o artigo visa contribuir para a literatura através de uma abordagem diferenciada, a qual, além de considerar a autocorrelação espacial a um nível de agregação que, até então, não era comum aos estudos a respeito do suicídio, também oferece um tratamento empírico diferenciado.

O artigo está organizado em mais quatro seções, além desta introdução: na segunda seção, apresenta-se uma revisão da literatura teórica e aplicada acerca do 
suicídio; na terceira detalha-se a estratégia empírica; na quarta são apresentados os resultados e discussões derivadas; e, por fim, na quinta elencam-se as principais conclusões do estudo.

\section{Revisão de Literatura}

Durkheim (2005) foi pioneiro a relacionar o suicídio com fatores macrossociais; segundo o autor, o incremento na renda, como também na escolaridade e nas taxas de divórcio, estaria diretamente relacionado ao aumento do suicídio. Nos dois primeiros casos, porque maiores níveis de renda e de instrução fomentariam o sentimento de independência, fazendo com que o individualismo prevalecesse.

Resultados nessa direção são encontrados por Chuang e Huang (1996) para os Estados Unidos. Quanto à escolaridade, Mitra e Shroff (2006) encontram relação positiva do suicídio com a taxa de alfabetização dos estados indianos, e a mesma relação foi confirmada para a população com curso superior e ensino médio nas províncias de Taiwan (LIN; LU, 2006).

Em contrapartida, para os Estados Unidos, as taxas de suicídio foram negativamente relacionadas com o nível de escolaridade (ABEL; KRUGER, 2005). Já o aumento dos divórcios favoreceria o suicídio, uma vez que romperia com o núcleo familiar. Em oposição a essa variável, está a taxa de fecundidade, já que o aumento do número de filhos representaria laços familiares mais solidificados. Crises, guerras e depressões contribuiriam para aumentar as probabilidades de ocorrerem suicídios na medida em que representariam riscos para a ordem social em vigor. Resultados semelhantes foram encontrados para países asiáticos, Irã e Itália (KYONNE, 2019; HAGHPARAST-BIDGOLI et al., 2018). Nesse sentido, a perda de status decorrente do desemprego seria um importante indutor do suicídio, especialmente entre a população do sexo masculino (SCHNEIDER et al., 2011).

Quanto à localidade, as taxas de suicídio tenderiam a ser maiores nas cidades do que no campo, tendo em vista que, no meio urbano, os laços sociais presumivelmente seriam mais efêmeros (DURKHEIM, 2005). Entretanto, Park e Lester (2012), em um estudo voltado à Coreia, encontram resultados divergentes. Segundo os autores, o maior índice de mortes autoprovocadas dá-se em áreas rurais e deve-se ao uso intensivo de pesticidas e produtos químicos. Fontanella et al. (2015) também encontram maiores taxas de suicídio em áreas rurais. Searles et al. (2014) consideram que as mortes no campo são maiores pelo fato de esses habitantes terem um acesso inadequado aos cuidados com saúde, enquanto Helbich et al. (2017) avaliam que o risco de suicídio é menor em regiões urbanas da Alemanha.

No que se refere à dispersão geográfica, as mortes por suicídio assumiriam um padrão altamente correlacionado em decorrência do efeito-contágio (DURKHEIM, 2005). Resultados de Abrutyn e Mueller (2014) sugerem que as re- 
lações sociais de imitação são um potencializador do suicídio. Em estudo voltado para adolescentes, os atores confirmaram que a ocorrência de suicídio em pessoas próximas, como amigos ou parentes, pode aumentar a vulnerabilidade de pensamentos e de tentativas suicidas nessa população. A relação das taxas de suicídio com a idade e com a renda postuladas pela ótica sociológica foi posteriormente investigada por Hamermesh e Soss (1974), numa perspectiva econômica.

Os principais resultados sugeriram um efeito negativo do aumento da renda em todas as idades, marcadamente entre a dos adultos jovens. Tal influência negativa da renda nas taxas de suicídio também é encontrada nos estudos de Lin e Lu (2006) para países asiáticos. Segundo Chen, Choy e Sawada (2009), em geral, melhores condições econômicas, como altos níveis de renda e maior crescimento econômico, atuam como um amortecedor das taxas de suicídio, enquanto a desigualdade de renda torna-se um importante fator de alavancagem.

A respeito da idade, os resultados de Hamermesh e Soss (1974) mostraram-se positivamente relacionados, evidenciando uma curva logística, o que é naturalmente explicado pelo fato de a utilidade vitalícia restante para os idosos ser logicamente pequena, ao passo que os jovens têm mais vida a perder. Por outro lado, a teoria econômica do suicídio não explica o aumento contínuo de mortes autoprovocadas entre os adolescentes. Nesse sentido, Cutler, Glaeser e Norberg (2001) conjecturam que, do ponto de vista da racionalidade econômica, é possível que mudanças na estrutura familiar ao longo do tempo, bem como alterações no ambiente social tenham elevado as taxas de desconto do futuro para a população mais jovem.

Outra explicação plausível é que os níveis médios de utilidade entre os jovens tenham reduzido em função do declínio do poder de compra das famílias ou em função do aumento da variância na distribuição de serviços públicos, o que colocaria mais jovens abaixo do nível de utilidade em que o suicídio se faz racional (CUTLER; GLAESER; NORBERG, 2001). Os efeitos do desemprego como consequência de recessões confirmaram-se, produzindo maiores impactos entre os jovens, mesmo para períodos de recessão não intensa.

Outros resultados apontam que a desigualdade e o valor relativo afetam mais os níveis de suicídio do que propriamente os valores absolutos da renda (DALY; WILSON; JOHNSON, 2012). Becker e Posner (2004) argumentam que as pessoas estão mais preocupadas com sua posição em relação a seus pares, o que justifica a relação positiva de medidas de desigualdade econômica com suicídio, como é caso dos achados de Gunnell et al. (1999) para a Inglaterra, de Andrés (2005) para países europeus e de Minoiu e Andrés (2008) para estados americanos. Em contrassenso, Leigh e Jencks (2007) não encontraram significância estatística da desigualdade de renda com os suicídios nos países desenvolvidos analisados. 
Conforme visto, as taxas de mortalidade por suicídio possuem uma dinâmica complexa que varia ao longo do tempo e conforme a localidade e a abrangência espacial estudada (WORLD HEALTH ORGANIZATION, 2015). Outrossim, é possível que o emprego de diferentes métodos altere a sensibilidade das variáveis de interesse. Além disso, há evidências de que fatores demográficos e socioeconômicos atingem de forma desigual os diferentes grupos de indivíduos (LOUREIRO; MOREIRA; SACHSIDA, 2013). No Brasil, as proporções continentais e a miscigenação etnocultural tornam o problema ainda mais sui generis, pois abriga áreas urbanas e rurais com populações de diferentes níveis econômicos e educacionais, o que reflete em diversos estilos de vida e pode influenciar diferentemente os fatores condicionantes do suicídio.

Tal diversidade é corroborada nos estudos de Souza, Minayo e Malaquias (2002) para as regiões metropolitanas brasileiras entre os anos de 1979 a 1998. Segundo os resultados, a incidência dos suicídios é dada em função dos diferentes espaços sociais, das faixas etárias, do sexo e dos meios utilizados para pôr fim à vida. A influência de variáveis econômicas nas taxas de suicídio foi testada em nível estadual por Shikida, Araújo Júnior e Gazzi (2006). O estudo no formato cross-section para o ano 2000 validou em grande parte as previsões teóricas e identificou uma relação não linear da idade com as taxas de suicídio. Do ponto de vista regional, taxas mais elevadas centraram-se nas regiões Sul e Centro-Oeste, sendo que o valor estimado para a região Sul ultrapassa o de todas as demais regiões. Esse fato indica possíveis efeitos advindos do clima ou de certas práticas agrícolas.

Nesse sentido, Benedito-Silva, Nogueira Pires e Calil (2007) atestam, através de séries temporais, o efeito sazonal nas mortes autoprovocadas, principalmente no Sul do país. Segundo evidências, há forte correlação dos suicídios com as temperaturas, bem como com o número de horas de sol (ORAVECZ et al., 2006). Por outro lado, Faria, Fassa e Meucci (2014) não descartam a influência do uso de agrotóxico e de pesticida nas taxas de suicídios, principalmente nas microrregiões onde predomina o cultivo de fumo e de algodão.

Outro estudo para os estados brasileiros, realizado por Loureiro, Mendonça e Sachsida (2010), investiga, por meio de um painel de dados, a relevância de variáveis econômicas como preditoras do suicídio. Como esperado, a renda reportou sinal negativo e o desemprego sinal positivo. Os resultados referentes à idade contradizem as expectativas do modelo de Hamermesh e Soss (1974), uma vez que não foi verificada estatisticamente a correlação positiva do suicídio com a população mais velha. Os resultados para a desigualdade mostraram-se diretamente relacionados ao suicídio, enquanto a pobreza revelou o inverso. Diante disso, uma importante conclusão dos autores corrobora as suposições de Becker e Posner (2004) de que a felicidade está mais atrelada ao consumo relativo dos indivíduos do que ao consumo absoluto. 
Nessa mesma perspectiva, Gonçalves, Gonçalves e Oliveira Júnior (2011) examinam os determinantes socioeconômicos do suicídio, dessa vez para as microrregiões brasileiras. Os resultados corroboram estudos anteriores de que a pobreza e o grau de ruralização são fatores explicativos do suicídio. O estudo inovou ao abordar aspectos espaciais sob a prerrogativa da existência de efeito-contágio. Tal efeito foi objeto do estudo de Loureiro, Moreira e Sachsida (2013) para os estados brasileiros nos anos de 1980 a 2009. Segundo os autores, a mídia é um importante meio propagador do suicídio entre homens jovens e figura entre os três principais motivadores para todos os grupos de pessoas, sendo antecedido apenas pelo desemprego e pela violência.

Machado, Rasella e Dos Santos (2015) investigam a influência da desigualdade de renda aliada a determinantes sociais com um painel de dados para os municípios brasileiros de 2000 a 2011. Os resultados revelaram que a desigualdade de renda é um importante preditor do suicídio, uma vez que o índice de Gini reportou sinal positivo. Por outro lado, a renda revelou-se um moderador, vista a relação negativa manifestada, atuando como fator de proteção contra o suicídio. Quanto às demais variáveis de cunho social (baixa ocupação, percentual de indivíduos desempregados e baixo nível de escolaridade), as taxas de suicídio mostraram-se positivamente relacionadas, sugerindo que situações de privação atuam como indutores do suicídio.

Tendo como pano de fundo o fato de que os suicídios são condicionados pelas características socioeconômicas, De Fraga, Massuquetti e Godoy (2017) aplicam uma regressão quantílica no exame da relação de tais características com a ocorrência de suicídios nos municípios brasileiros e do Rio Grande do Sul. Os principais resultados indicam que a pobreza é um importante preditor das mortes autoprovocadas, uma vez que se mostrou significativa e positivamente relacionada às mortes autoprovocadas em todos os quantis estimados. Quanto aos indicadores socioeconômicos, a relação foi confirmada apenas para os municípios brasileiros como um todo, dado que, para os municípios gaúchos, as variáveis relacionadas à saúde e à educação não reportaram significância estatística.

De modo geral, os achados empíricos vão ao encontro da teoria sociológica e econômica do suicídio, atestando que sua explicação deve ser buscada tanto nas condições sociais do meio quanto em fatores puramente econômicos. Entretanto, a falta de consenso entre as questões atreladas à idade, à dicotomia urbano-rural e ao papel da renda, seja ela absoluta ou relativa, abre espaço para novas investigações. Soma-se a isso a singularidade de estudos que admitem a interação espacial nas análises dessa problemática, destacando-se os resultados de Gonçalves, Gonçalves e Oliveira Júnior (2011), Siqueira (2017) e Amaral (2018), os quais reforçam a importância do espaço na determinação do fenômeno. 
É nesse contexto de controvérsia, em termos de resultado e de escassez, e em termos de estudos espacialmente aplicados, que se insere o presente trabalho, o qual também se propõe a considerar efeitos decorrentes da autocorrelação espacial nas mortes por suicídio. Entretanto, diferentemente dos estudos citados, este tem como diferencial a utilização de variáveis ao nível de municípios e avança metodologicamente ao contribuir com uma especificação espacial de modelo tobit, a qual modela a forte incidência de zeros na variável resposta como variável censurada.

\section{Método}

Para responder ao problema de pesquisa e atingir o objetivo proposto, visando uma melhor compreensão do fenômeno, a análise exploratória de dados espaciais (Aede) antecede os procedimentos econométricos. Através da Aede é possível, mediante mapas temáticos, visualizar a distribuição dos dados no território e, com o uso da estatística espacial, identificar padrões de associação no espaço, bem como instabilidades e observações atípicas, as quais podem ser diagnosticadas mediante o emprego complementar de estatísticas espaciais apropriadas.

\subsection{Análise Exploratória de Dados Espaciais}

A Aede é um conjunto de técnicas que utiliza estatística espacial e geoestatística para apreender representações das características dos dados no espaço e, a partir daí, pode-se detectar efeitos espaciais condizentes com relação de dependência e/ou de heterogeneidade espaciais (BIVAND, 2010). Tais efeitos são inicialmente investigados por meio de testes de autocorrelação global, aqui especificamente a estatística I de Moran, e indicadores locais de associação espacial (Lisa), aqui especificamente a estatística I de Moran local.

Ambos os testes têm como hipótese nula a aleatoriedade espacial, de modo que, por um lado, valores estatisticamente superiores ao valor esperado indicam autocorrelação espacial positiva, apontando para similaridade espacial. Por outro lado, valores estatisticamente inferiores ao valor esperado denotam uma autocorrelação espacial negativa, sugerindo dissimilaridades entre a região de referência e a vizinhança. Algebricamente, a representação do I de Moran é dada por:

$$
I=\frac{n \sum \sum \boldsymbol{w}_{i j}\left(y_{i}-\bar{y}\right)\left(y_{j}-\bar{y}\right)}{\sum \sum \boldsymbol{w}_{i j} * \sum\left(y_{i}-\bar{y}\right)^{2}}
$$


em que:

$n$ : número de unidades geográficas;

$y_{i, j}$ : valores da variável de interesse no local de referência, seja este i ou j;

$\bar{y}$ : média dos valores da variável de interesse na vizinhança;

$w_{i j}$ : matriz de pesos espaciais indicando a vizinhança entre $i$ e $j$.

A Estatística I de Moran é o indicador global mais utilizado para detectar a autocorrelação espacial, entretanto, a rejeição da hipótese nula não permite discernir se a autocorrelação detectada é decorrente da heterogeneidade ou da dependência espacial (ANSELIN; FLORAX, 1995). Assim, para refinar a análise da interação espacial, utiliza-se a estatística I de Moran local, a qual deriva de uma adaptação do teste global, conforme (ANSELIN, 1995):

$$
I_{i}=z_{i} \sum_{j} w_{i j} z_{j}
$$

em que:

$z_{i}$ e $z_{j}$ : desvios em torno da média das observações;

$w_{i j}$ : matriz de pesos espaciais indicando a vizinhança entre $i$ e j.

Sob a rejeição da hipótese nula (território espacialmente homogêneo), o teste aponta para a existência de agrupamentos locais com características próprias. Uma das representações usuais desses testes é através do gráfico de dispersão, em que o desvio dos atributos em relação à média é retratado no eixo das abscissas, enquanto o valor da média dos vizinhos aparece no eixo das ordenadas. Cada quadrante do gráfico de dispersão representa um dos quatro padrões de associação espacial local entre a região de referência e as adjacências. No primeiro e no terceiro quadrantes, localizam-se padrões de autocorrelação positiva alto-alto ou baixo-baixo, respectivamente, os quais revelam a formação de clusters similares, já o segundo e o quarto quadrantes abrigam a autocorrelação negativa, baixo-alto ou alto-baixo, respectivamente, indicando a presença de outliers espaciais.

\subsection{Procedimentos Metodológicos}

O presente estudo tem como unidades de análises os municípios da Federação no ano de 2010), sendo que a variável de interesse é a taxa de suicídio, coletada a partir dos códigos da Classificação Internacional de Doenças (CID), junto ao Sistema de Informações de Mortalidade (SIM) do Ministério da Saúde, através do Datasus. A agregação das variáveis confere uma abordagem ecológica, já que 
o foco deixa de ser o sujeito e passa a ser a população. Tal estratégia impossibilita que inferências sejam interpretadas como uma generalização do comportamento de indivíduos, entretanto os resultados a nível municipal são desejáveis, pois é dessa forma que o sistema público de saúde brasileiro, com base na Norma Operacional de Assistência à Saúde (MINISTÉRIO DA SAÚDE, 2001), implementa ações regionais de atenção à saúde (GONÇALVES; GONÇALVES; OLIVEIRA JÚNIOR 2011; AMARAL 2018; ANSELIN, 2011).

As informações disponibilizadas pelo SIM são compilações das declarações de óbito (DO), que, segundo disposição legal, devem ser preenchidas por um médico do Instituto Médico Legal quando a causa da morte for externa. No caso dos municípios que não dispõem de um instituto, as autoridades judiciais nomeiam um médico sobre o qual recai a responsabilidade de autuar o motivo da morte. Para determinar que a morte é um suicídio, precisa-se identificar a intenção de morrer, o que nem sempre é claro e fica ainda mais difícil na ausência de especialistas. Consequentemente, é provável que alguma parcela de mortes autoprovocadas seja registrada como acidente de trânsito de um único veículo, envenenamento não intencional ou causa indeterminada (BOTEGA, 2014).

Apesar de a qualidade dos dados do SIM ter evoluído muito a partir dos anos 2000, quando houve considerável valorização sobre o uso das estatísticas vitais, contribuindo para a melhor cobertura e confiabilidade das informações, a subnotificação continua sendo uma realidade, principalmente nos municípios mais precários (FRIAS; SZWARCWALD; LIRA, 2014; ALMEIDA; SZWARCWALD, 2017). Somam-se aos fatores já mencionados razões de cunho religioso, cultural e judicial que também contribuem para distorções e para o sub-registro, uma vez que em muitas comunidades o suicídio ainda é tabu, além de ser considerado crime no Brasil pelo Código Penal, podendo resultar em negativa de ressarcimento em caso de planos de saúde ou seguros de vida.

O fato de o suicídio ser um fenômeno de ocorrência rara resulta em uma amostra demasiadamente composta por zeros, principalmente quando se trata de escalas espaciais mais desagregadas, já que muitos municípios não registram ocorrências no referido ano (XU; LEE, 2015). Metodologicamente, sabe-se que forte presença de zeros compromete as pressuposições do modelo clássico de regressão linear (MQO), requerendo técnicas apropriadas de tratamento. Entre as possibilidades mais comuns para lidar com essa questão está o emprego de modelos para dados de contagem, a agregação em unidades territoriais maiores, bem como a utilização dos dados na forma de somatório ou de média para um longo período de tempo, conforme os estudos desenvolvidos por Gonçalves, Gonçalves e Oliveira Júnior (2011), Siqueira (2017), De Fraga, Massuquetti e Godoy (2017) e Amaral (2018). 
Tais técnicas não garantem boas propriedades para os estimadores quando a variável dependente aparece concentrada em pontos iguais a um valor limite, produzindo resultados tendenciosos e inconsistentes, pois uma parcela considerável dos dados, frequentemente não negativos, é latente e, na maior parte dos casos, é atribuído a eles o valor nulo (AMEMIYA, 1973). Isso porque a distribuição das taxas de suicídio é limitada à esquerda, uma vez que não é possível ter valores menores que zero. Todavia, a distribuição é contínua em seus valores positivos até o valor unitário, que, apesar de estatisticamente possível, é altamente improvável e não é encontrado empiricamente. Modelos de regressão censurados ou modelos tobit podem ser aplicados quando a variável de interesse é censurada, ou seja, seus valores não são observáveis além de um determinado limite.

O modelo tobit, desenvolvido por James Tobin (1958), estima a relação entre uma variável não negativa e variáveis independentes. Supõe que existe uma variável latente ou não observável $y^{*}$. A variável latente $y^{*}$ é considerada linearmente relacionada com as variáveis independentes $(X)$ mais um termo de erro, que tem como objetivo capturar influências aleatórias.

A equação estrutural do modelo tobit é:

$$
\boldsymbol{y}^{*}=\boldsymbol{X}^{\prime} \beta+\boldsymbol{u}
$$

em que:

$\boldsymbol{x}$ : matriz $k \times n$ de variáveis explicativas;

$\beta$ vetor $k \times 1$ de coeficientes;

$\boldsymbol{u}$ : vetor $n \times 1$ de erros aleatórios normalmente distribuídos.

A partir do modelo genérico, a lógica da especificação tobit admite que a variável latente $y_{i}^{*}$ é observada somente quando um valor limite "c" é excedido de tal forma que a variável dependente passa a ser expressa como:

$$
y_{i}=\left\{\begin{array}{lll}
y_{i}^{*}, & \text { se } & y_{i}^{*}>c \\
0, & \text { se } & y_{i}^{*} \leq c
\end{array}\right.
$$

Com isso, as observações iguais ou menores que o valor limite acabam sendo reportadas pelo próprio valor limite. Sabendo-se que $\phi$ representa a função de densidade acumulada e $\varnothing$ constitui a função de densidade de probabilidade da distribuição normal padrão, sob a suposição de normalidade, representa-se o modelo tobit por: 


$$
y_{i}=\sigma \boldsymbol{\Phi}\left(\frac{x_{i} \beta}{\sigma}\right)\left[\frac{x_{i} \beta}{\sigma}+\frac{\phi\left(\frac{x_{i} \beta}{\sigma}\right)}{\boldsymbol{\Phi}\left(\frac{x_{i} \beta}{\sigma}\right)}\right]+u_{i}
$$

Para a especificação indicada, o termo de erro aleatório é independente e identicamente distribuído, entretanto tal suposição pode não ser verificada tratando-se de estudos com dados espaciais. Caso o termo de erro seja espacialmente correlacionado, as estimativas do modelo podem ser inconsistentes (FLEMING, 2004). Logo, a investigação e o posterior tratamento da presença de autocorrelação espacial se fazem relevantes.

Com base na literatura apresentada e no intuito de captar efeitos espaciais, assume-se especificação com defasagem espacial da variável latente dependente, que passa a ser representada como:

$$
\boldsymbol{y}^{*}=\rho \boldsymbol{W} \boldsymbol{y}^{*}+\boldsymbol{X}^{\prime} \beta+\boldsymbol{u}
$$

em que:

$\rho$ parâmetro de defasagem espacial;

$W$ matriz $n \times n$ de pesos espaciais, definida, aqui, a partir de contiguidade espacial, isto é, rainha de primeira ordem que considera como vizinhos todos os municípios que possuem uma fronteira ou vértice em comum.

O parâmetro de defasagem espacial reflete a autocorrelação espacial, ao passo que a introdução da matriz de pesos espaciais possibilita captar, nessa especificação, a abrangência do efeito espacial entre as unidades. Assim, a variável dependente de uma localidade passa a ser também determinada pelas características da vizinhança, além das suas características próprias na forma de variáveis explicativas, tipificando a ocorrência de transbordamentos espaciais. ${ }^{1}$

Na forma reduzida, segundo Amaral e Anselin (2014), o modelo de defasagem espacial para variáveis latentes é reescrito como:

$1 \quad$ Dentre os modelos espaciais mais conhecidos estão os do tipo spatial autorregressive model (SAR) e spatial error model (SEM) - ou modelo de defasagem espacial e modelo de erro espacial. No modelo SAR, a correlação espacial está presente na inclusão da defasagem espacial da variável dependente como variável explicativa, enquanto, no modelo SEM, os erros são considerados como espacialmente correlacionados. Há ainda especificações mistas que podem ser vistas com mais detalhes em LeSage e Pace (2009). Uma importante consequência da presença de defasagem espacial são os efeitos de retroalimentação. Ou seja, a variação nas variáveis independentes de uma localidade qualquer impacta não somente a variável dependente da própria localidade (efeito direto), mas também as variáveis dependentes das unidades vizinhas (efeito indireto). A soma desses impactos constitui os efeitos totais, conforme exemplificado por LeSage e Pace (2009). 


$$
\boldsymbol{y}^{*}=(\boldsymbol{I}-\rho \boldsymbol{W})^{-1} \boldsymbol{X}^{\prime} \beta+(\boldsymbol{I}-\rho \boldsymbol{W})^{-1} \boldsymbol{u}
$$

Uma implicação da especificação de defasagem espacial para o modelo tobit é que a presença do termo inverso acarreta uma distribuição normal multivariada truncada, o que torna computacionalmente demandante a obtenção da função de máxima verossimilhança, de modo que não se encontram na estatística clássica ferramentas tão eficazes quanto na abordagem bayesiana (GOLGHER, 2015).

Basicamente, os valores censurados são estimados condicionando-os às observações sem censura. Como se trata de um modelo espacial, a obtenção da distribuição a posteriori não se dá de forma direta, visto que a resolução analítica desses modelos é demasiadamente complexa em função da interdependência entre as observações. Para viabilizar tal processo, utiliza-se o amostrador de Gibbs, o qual consiste em uma simulação estocástica via cadeia de Markov (MCMC). Para tanto, fez-se uso do pacote spatial probit, implantado por Wilhelm e De Matos (2013) no software R. ${ }^{2}$

\subsection{Variáveis e Banco de Dados}

No intuito de contemplar os preceitos teóricos inerentes ao suicídio elencados na primeira seção do trabalho, as variáveis exploratórias do modelo abrangem aspectos econômicos, sociais e demográficos mediante a inserção da renda, índice de Gini, índice de desenvolvimento humano municipal (IDHM) da educação, grau de formalização do trabalho, taxa de fecundidade, população urbana e total da área plantada das culturas de fumo e de algodão. A última é empregada no modelo como proxy para organofosforados, com a finalidade de captar possíveis efeitos desses agrotóxicos no aumento das taxas de suicídio.

Usualmente, a literatura emprega a variável desemprego para captar efeitos decorrentes do mercado de trabalho sobre o suicídio, tais como o peso da perda salarial sobre a decisão de se suicidar, mas a existência de programas de seguro desemprego pode enfraquecer o efeito final sobre o suicídio. Assim, utiliza-se alternativamente o grau de formalização do trabalho. O Quadro 1 apresenta uma síntese das variáveis utilizadas, bem como de sua descrição e fonte.

2 Para a especificação de modelos espaciais de variáveis discretas, segue-se a recomendação de Golgher (2015). Foram utilizadas 1000 MCMC interações, com burn-in de 100 interações e a definição da apriorística por $\rho \sim \operatorname{Beta}(1,1)$ e $\beta \sim N(0,1)$. 


\begin{tabular}{|c|c|c|}
\hline Variável & Descrição & Fonte \\
\hline Taxa de fecundidade & $\begin{array}{l}\text { Número médio de filhos que uma mulher deverá ter ao terminar } \\
\text { o período reprodutivo (de } 15 \text { a } 49 \text { anos de idade). }\end{array}$ & Atlas PNUD \\
\hline Índice de Gini & $\begin{array}{l}\text { Mede o grau de desigualdade existente na distribuição de } \\
\text { indivíduos segundo a renda domiciliar per capita. Seu valor varia } \\
\text { de zero, quando não há desigualdade (a renda domiciliar per } \\
\text { capita de todos os indivíduos tem o mesmo valor), a um, quando } \\
\text { a desigualdade é máxima (apenas um indivíduo detém toda a } \\
\text { renda). O universo de indivíduos é limitado àqueles que vivem em } \\
\text { domicílios particulares permanentes. }\end{array}$ & Atlas PNUD \\
\hline Renda per capita & $\begin{array}{l}\text { Razão entre o somatório da renda de todos os indivíduos } \\
\text { residentes em domicílios particulares permanentes e o número } \\
\text { total desses indivíduos. Valores em reais de } 1^{\circ} \text { de agosto de } 2010 \text {. }\end{array}$ & Atlas PNUD \\
\hline $\begin{array}{l}\text { Grau de formalização } \\
\text { do trabalho das } \\
\text { pessoas ocupadas }\end{array}$ & $\begin{array}{l}\text { Razão entre o número de pessoas de } 18 \text { anos ou mais } \\
\text { formalmente ocupadas e o número total de pessoas ocupadas } \\
\text { nessa faixa etária multiplicado por } 100 \text {. Foram considerados como } \\
\text { formalmente ocupados os empregados com carteira de trabalho } \\
\text { assinada, os militares do exército, da marinha, da aeronáutica, } \\
\text { da polícia militar ou do corpo de bombeiros, os empregados } \\
\text { pelo regime jurídico dos funcionários públicos, assim como os } \\
\text { empregadores e trabalhadores por conta própria que eram } \\
\text { contribuintes de instituto de previdência oficial. }\end{array}$ & Atlas PNUD \\
\hline População urbana & População residente na área urbana. & Atlas PNUD \\
\hline $\begin{array}{l}\text { Índice de } \\
\text { desenvolvimento } \\
\text { humano municipal - } \\
\text { dimensão educação } \\
\text { (IDHM) }\end{array}$ & $\begin{array}{l}\text { Índice sintético da dimensão educação, que é um dos três } \\
\text { componentes do IDHM. É obtido através da média geométrica } \\
\text { do subíndice de frequência de crianças e de jovens à escola, com } \\
\text { peso de dois terços, e do subíndice de escolaridade da população } \\
\text { adulta, com peso de um terço. }\end{array}$ & Atlas PNUD \\
\hline $\begin{array}{l}\text { Área plantada de fumo } \\
\text { e de algodão }\end{array}$ & Somatório das áreas plantadas que cultivam fumo e algodão. & IBGE Sidra \\
\hline
\end{tabular}

Fonte: Elaboração própria a partir de Atlas PNUD (2010) e IBGE Sidra (IBGE, 2010).

Além das fontes citadas no Quadro 1, também se recorreu ao SIM via Datasus para se capturar os dados do suicídio, variável dependente, que foram extraídos na forma de ocorrências e convertidos em taxa por 100.000 mil habitantes. Com isso, espera-se que o efeito das variáveis econômicas, sociais e demográficas sobre as taxas de suicídio seja confirmada, bem como a interação espacial do fenômeno, e que a proxy para agrotóxicos exerça influência significativa. Em posse da apuração empírica dos fatores que motivam o suicídio nos municípios brasileiros, pretende-se fornecer as bases para o desenho de políticas de contenção e de prevenção mais específicas à realidade do país, a qual não tem recebido a devida atenção dos órgãos governamentais. 


\section{Resultados}

Na presente seção são apresentados os resultados da Aede, seguida dos procedimentos econométricos. Conforme abordado anteriormente, a análise é composta pelos 5.565 municípios brasileiros, dos quais 2.864 não registraram ocorrências de suicídio no ano de 2010, o que reafirma a raridade do fenômeno, além da possível incidência de sub-registro já apontada. A Figura 1 plota a distribuição das taxas de suicídio por 100 mil habitantes e facilita a observação da dispersão dessa variável, a qual aparece visivelmente mais concentrada nos municípios das regiões Sul e Sudeste do Brasil.

Figura 1 - Box-plot e distribuição da taxa de suicídio por 100 mil habitantes nos municípios brasileiros (2010) Fonte: Elaboração própria a partir dos resultados da pesquisa.

(a)

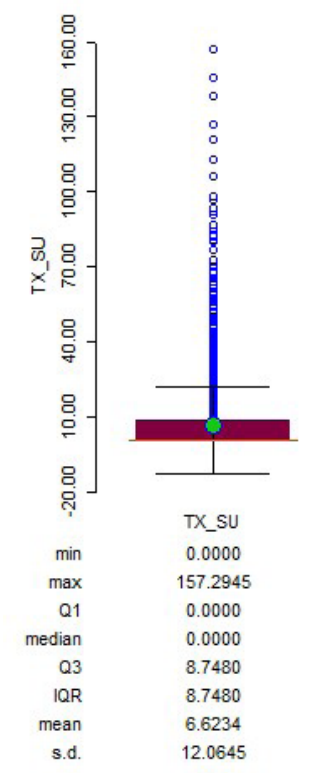

(b)

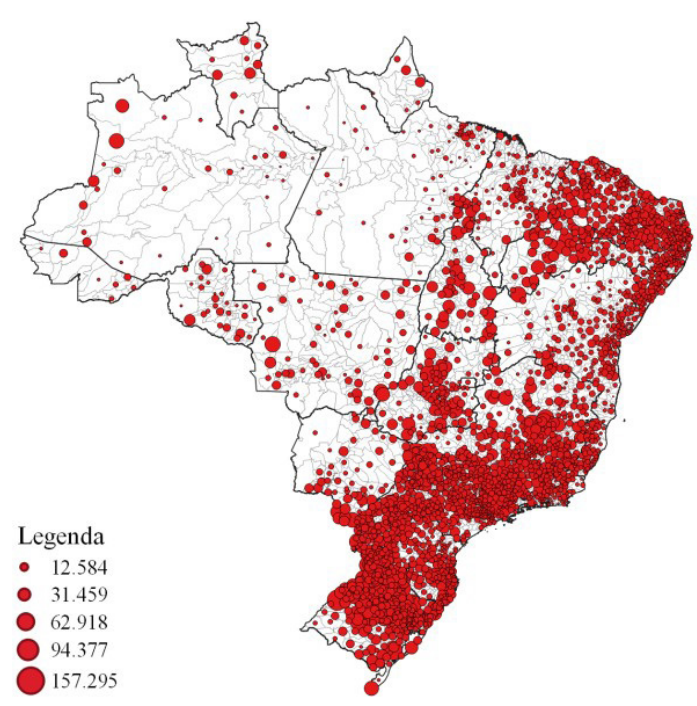

Fonte: Elaboração própria a partir dos resultados da pesquisa.

De acordo com o box-plot, a média observada para os municípios é de 6,623 suicídios para cada 100 mil habitantes, com um desvio padrão de 12,065. O valor mínimo foi encontrado em mais de 51\% dos municípios, enquanto o valor máximo - 157,295 suicídios para cada 100 mil habitantes - foi reportado pelo município de Santa Rita D’Oeste, no estado de São Paulo. A mediana é diferente da média 
e igual ao primeiro quartil, revelando que a distribuição dos dados para a taxa de suicídio é assimétrica e positiva. A amplitude interquartílica encontra-se entre zero e 8,748 suicídios por 100 mil habitantes, sugerindo uma pequena dispersão. Por outro lado, a forte concentração da amostra em torno de zero faz com que uma parte considerável de taxas superiores seja considerada como observações discrepantes. Assim, 443 municípios representam outliers, dos quais aproximadamente $30 \%$ estão localizados no Rio Grande do Sul. Questões acerca da espacialidade dos dados são abordadas na Figura 2.

Figura 2 - I de Moran global e local da taxa de suicídio por 100 mil habitantes nos municípios brasileiros (2010)

(a)

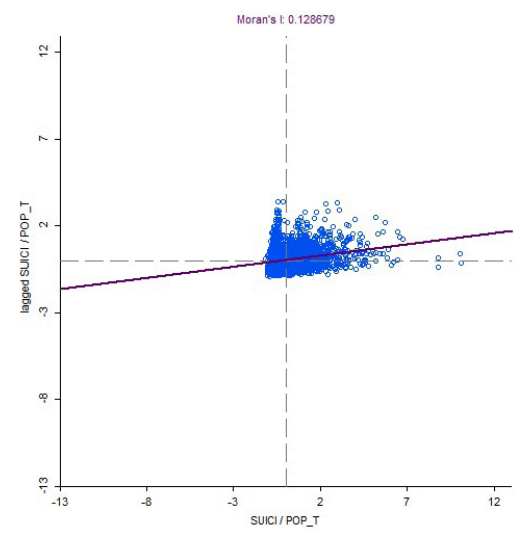

(b)

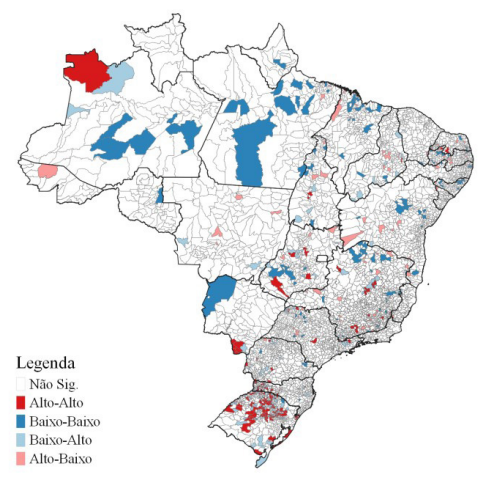

Fonte: Elaboração própria a partir dos resultados da pesquisa.

Nota: O I de Moran global foi avaliado a partir de 999 permutações com pseudo $p$-valor $=$ 0,001 .

Segundo a Figura 2, parte $a$, é perceptível a presença de associação global espacial da mortalidade por suicídio nos municípios brasileiros. Trata-se de uma associação espacial positiva e estatisticamente significativa, ainda que seu valor absoluto seja baixo. O valor da estatística (0.128) gerado a partir de 999 permutações rejeitou a hipótese nula de aleatoriedade a um pseudo $p$-valor de 0,001.

Isso fornece indícios de que os municípios dotados de taxas acima da média são, em geral, cercados por uma vizinhança com taxas médias nas mesmas condições. O mesmo pode-se dizer dos municípios com valores abaixo da média. Tal padrão de similaridade espacial foi diagnosticado com base em uma matriz de pesos do tipo rainha de primeira ordem, a qual considera como vizinhos todos os munícipios que possuem uma fronteira ou vértice em comum. 
Aglomerados específicos são retratados na Figura 2, parte $b$. A maioria dos municípios, cerca de 4.940, não apresentou significância estatística para os indicadores de autocorrelação local. Resultados nessa direção apareceram nos estudos de Amaral (2018). Chama atenção a elevada concentração da tipologia alto-alto entre os municípios sulinos, sobretudo no estado gaúcho. Em contrapartida, a tipologia baixo-baixo aparece distribuída ao longo do território brasileiro, manifestando-se sutilmente com maior frequência entre os municípios do Norte e Nordeste. Dada a diferença existente entre a porção meridional e o restante do país, Siqueira (2017), em seu estudo para microrregiões, identificou a ocorrência de "dois Brasis".

Apesar das evidências, os resultados exploratórios não permitem conclusões definitivas acerca da mortalidade por suicídio, tendo em vista a complexidade do fenômeno e o fato de que os dados são amiúde afetados pela qualidade dos registros, a qual é regionalmente desigual (FRIAS et al., 2008). Assim, é possível que muitas localidades que reportam aglomerados baixo-baixo não tenham necessariamente taxas de suicídios menores, mas, sim, sofram com a precariedade dos registros de mortalidade, situação comum entre os municípios mais carentes e remotos do Norte e do Nordeste (ANDRADE; SZWARCWALD, 2007). Em contrapartida, a omissão de registros tende a ser reduzida nas regiões que dispõem de melhor infraestrutura. Esse pode ser o caso dos municípios sulinos em que as taxas de suicídios são alavancadas pela maior cobertura dos registros de mortalidade, visto o acesso facilitado a bens e a serviços públicos.

Em posse da análise exploratória de dados espaciais, dá-se sequência à exposição dos resultados das estimações econométricas, que tem como objetivo explicar a relação do suicídio com fatores socioeconômicos dos municípios brasileiros. Para tanto, leva-se em consideração a existência da censura e da dinâmica espacial na variável dependente decorrente da interação com regiões adjacentes. Nesse sentido, foi estimado o modelo SAR tobit, conforme reportado na Tabela 1, juntamente com os resultados de modelo de mínimos quadrados ordinários (MQO) e tobit não espacial (TOBIN, 1958).

Tabela 1 - Resultados das estimações: variável dependente: taxa de suicídio

\begin{tabular}{lcccccc}
\hline Variáveis & a) MQO & & b) Tobit & \multicolumn{3}{c}{ c) SAR tobit } \\
\hline Intercepto & -7.814 & $*$ & -65.533 & $* * *$ & -63.782 & $* * *$ \\
& $(3.754)$ & & $(7.505)$ & & $(7.903)$ & \\
Gini & -5.299 & & -8.240 & & -7.872 & \\
& $(3.221)$ & & $(6.311)$ & & $(6.120)$ & \\
IDMH_educ & -6.760 & $*$ & -19.670 & $* *$ & -18.814 & $* * *$ \\
& $(3.194)$ & & $(6.330)$ & & $(6.738)$ & \\
Ln renda & 5.501 & $* * *$ & 11.184 & $* * *$ & 10.608 & $* * *$ \\
\hline
\end{tabular}

Continua... 
Conclusão.

\begin{tabular}{|c|c|c|c|c|c|c|}
\hline \multirow{2}{*}{ Variáveis } & \multicolumn{2}{|l|}{ a) MQO } & \multicolumn{2}{|l|}{ b) Tobit } & \multicolumn{2}{|c|}{ c) SAR tobit } \\
\hline & (0.698) & & (1.383) & & $(1.492)$ & \\
\hline \multirow[t]{2}{*}{ P_formal } & -0.036 & $*$ & -0.094 & $* *$ & -0.097 & $* * *$ \\
\hline & $(0.016)$ & & $(0.032)$ & & $(0.033)$ & \\
\hline \multirow[t]{2}{*}{ Ln_fumal } & 0.418 & $* * *$ & 0.852 & $* * *$ & 0.768 & $* * *$ \\
\hline & $(0.077)$ & & $(0.145)$ & & (0.139) & \\
\hline \multirow[t]{2}{*}{ Tx fecundidade } & -0.762 & & -2.143 & $*$ & -1.875 & $* *$ \\
\hline & $(0.426)$ & & $(0.859)$ & & $(0.846)$ & \\
\hline \multirow[t]{2}{*}{ Ln_pop_urb } & -1.104 & $* * *$ & 2.006 & $* * *$ & 2.095 & $* * *$ \\
\hline & $(0.138)$ & & $(0.273)$ & & $(0.246)$ & \\
\hline I-Moran & 0.036 & & 0.088 & $* * *$ & & \\
\hline \multirow[t]{2}{*}{ Sige } & & & & & 413.348 & $* * *$ \\
\hline & & & & & (13.719) & \\
\hline \multirow[t]{2}{*}{ Rho } & & & & & 0.118 & $* * *$ \\
\hline & & & & & $(0.026)$ & \\
\hline
\end{tabular}

Fonte: Elaboração própria a partir dos resultados da pesquisa.

Nota: Erros padrões entre parênteses; significância: *p<0.10, ${ }^{* *} p<0,05,{ }^{* * *} p<0,01$.

Observa-se que os coeficientes reportados pelo MQO são claramente subestimados, representando um viés sistemático nas estimativas que ignoram a censura. O I de Moran, calculado a partir dos resíduos generalizados do modelo tobit (AMARAL; ANSELIN, 2014), rejeita a hipótese nula de aleatoriedade espacial a um $p$-valor de 0,001, indicando que a autocorrelação espacial de 0,118 é significativa. A Tabela 1 também atesta que o modelo tobit apresenta magnitudes absolutas ligeiramente maiores em face da sua versão defasada espacialmente, mas que não são estatisticamente diferentes, provavelmente devido ao baixo valor absoluto do parâmetro de defasagem espacial. Variáveis como IDHM dimensão educação, grau de formalização do trabalho e taxa de fecundidade tornaram-se mais significativas nessa última estimação.

Cabe atentar para a ausência de significância estatística do índice de Gini nos três casos e para a reiterada importância da renda per capita, tamanho da população urbana e área plantada na explicação das taxas de suicídio. Ademais, o coeficiente autorregressivo para o modelo espacial certifica a defasagem espacial da variável dependente, assinalando que os municípios com altas taxas de suicídio, mesmo controlando os atributos socioeconômicos e demográficos, tendem a se encontrar agrupados espacialmente.

A presença significativa do parâmetro de defasagem espacial indica a existência de um efeito multiplicador. Em um modelo linear clássico, o efeito marginal de uma mudança no valor de uma variável explicativa contínua é dado por 
$\partial y_{i} / \partial x_{i}=\beta$. Em um modelo de defasagem espacial, isso não mais se aplica e o efeito marginal passa a ser $\frac{\partial y_{i}}{\partial x_{i}}=(\mathrm{I}-\rho W)^{-1} \beta$. Esse efeito marginal é comumente chamado de efeito total. Por sua vez, o efeito marginal ignorando a associação espacial é chamado de efeito direto. Chama-se, então, de efeito indireto a diferença entre ambos. Com base no exposto, os resultados são apresentados na Tabela 2.

Tabela 2 - Resultados das estimações: variável dependente: taxa de suicídio

\begin{tabular}{|c|c|c|c|c|}
\hline \multirow{2}{*}{ Variável } & \multirow[b]{2}{*}{ Abaixo de 0,05} & \multicolumn{3}{|c|}{ SAR tobit } \\
\hline & & \multicolumn{2}{|c|}{ Média da distribuição } & \multirow[t]{2}{*}{ Acima de 0,95} \\
\hline \multicolumn{4}{|c|}{ a) Efeito direto } & \\
\hline Gini & -8.736 & -3.915 & & 1.129 \\
\hline IDMH_educ & -14.480 & -9.356 & $* * *$ & 4.169 \\
\hline Ln renda & 4.073 & 5.275 & $* * *$ & 6.634 \\
\hline$P_{-}$formal & -0.074 & -0.048 & $* * *$ & -0.018 \\
\hline Ln_fumal & 0.270 & 0.382 & $* * *$ & 0.479 \\
\hline Txfecund. & -1.544 & -0.932 & $* *$ & -0.223 \\
\hline Ln_pop_urb & 0.812 & 1.042 & $* * *$ & 1.236 \\
\hline \multicolumn{5}{|c|}{ b) Efeito indireto } \\
\hline Gini & -26.187 & -11.737 & & 3.374 \\
\hline IDMH_educ & -43.475 & -28.054 & $* * *$ & -12.521 \\
\hline Ln renda & 12.214 & 15.817 & $* * *$ & 19.902 \\
\hline$P_{-}$formal & -0.222 & -0.144 & $* * *$ & -0.054 \\
\hline Ln_fumal & 0.130 & 1.145 & $* * *$ & 1.433 \\
\hline Txfecund. & 0.811 & -2.796 & $* *$ & -0.670 \\
\hline Ln_pop_urb & 0.413 & 3.123 & $* * *$ & 3.707 \\
\hline \multicolumn{5}{|c|}{ c) Efeitos totais } \\
\hline Gini & -34.923 & -15.652 & & 4.503 \\
\hline IDMH_educ & -57.955 & -37.410 & $* * *$ & -16.689 \\
\hline Ln renda & 16.287 & 21.092 & $* * *$ & 26.536 \\
\hline P_formal & -0.295 & -0.192 & $* * *$ & -0.073 \\
\hline Ln_fumal & 1.080 & 1.527 & $* * *$ & 1.913 \\
\hline Txfecund. & -6.164 & -3.728 & $* *$ & -0.893 \\
\hline Ln_pop_urb & 3.251 & 4.165 & $* * *$ & 4.943 \\
\hline
\end{tabular}

Fonte: Elaboração própria a partir dos resultados da pesquisa.

Nota: Erros padrões entre parênteses; significância: ${ }^{*} p<0.10,{ }^{* *} p<0,05,{ }^{* * * *} p<0,01$.

Observa-se que o IDHM dimensão educação exerce uma influência composta de -37,410 pontos sobre a variável dependente. Como esse índice varia de zero a um, isso significa que o aumento de 0,01 ponto está associado à redução de 0,374 nas mortes por suicídios a cada 100 mil habitantes, o que é um aspecto 
particularmente relevante para a explicação do suicídio, de modo que, quanto melhores os índices educacionais, menores são as taxas de mortes voluntárias.

Esse resultado, ainda que na contramão da teoria sociológica na qual previa o encorajamento da sensação de liberdade e, por conseguinte a degeneração do sentimento de solidariedade entre os pares como consequência de incrementos na instrução, parece estar na sociedade moderna atrelado à redução da vulnerabilidade social (GONÇALVES; GONÇALVES; OLIVEIRA JÚNIOR, 2011). Tal argumento é factível com a Teoria do Capital Humano, amplamente difundida na ciência econômica, a qual reconhece na escolaridade um dos instrumentos fundamentais para contrapor as restrições socioeconômicas da população. Além disso, a perspectiva de maior renda futura, decorrente da maior escolaridade, eleva a utilidade do tempo de vida restante, diminuindo o incentivo ao suicídio, conforme previsto por Hamermesh e Soss (1974).

A renda per capita dos municípios exerce um efeito total de 21,092 sobre as taxas de suicídio, indicando que o aumento de 1\% nessa variável está associado a um aumento de 0,211 ponto na taxa de suicídio. Daquele montante, 0,053 corresponde a efeitos diretos e os outros 0,158, a efeitos indiretos. A relação positiva entre suicídio e renda per capita, mesmo que controversa na literatura, vai ao encontro do pensamento durkheimiano, segundo o qual incrementos na renda atuariam como dissipadores dos laços sociais. Outra explicação a se considerar é que o crescimento da renda nem sempre é garantia de um aumento de bem-estar para a população, especialmente nos países em desenvolvimento, como é o caso do Brasil, em que o crescimento do PIB é acompanhado de elevada concentração no topo da pirâmide, fazendo com o que o número de suicídios cresça entre aqueles que se situam na base (DE FRAGA; MASSUQUETTI; GODOY, 2017).

Por sua vez, o grau de formalização do trabalho exerce um efeito total de $-0,192$, ou seja, para cada ponto percentual adicional nessa variável, a taxa de suicídios tende a reduzir-se em 0,192 ponto, sendo 0,048 referente aos efeitos diretos e 0,144 aos efeitos indiretos. Esse resultado revela o papel dos vínculos formais de trabalho como fator de proteção ao suicídio na medida em que favorecem não só a estabilidade financeira como também propiciam acesso ao Estado de bem-estar social, além de minorar o sentimento de marginalidade, reafirmando a identidade social no ambiente capitalista (SANTOS et al., 2011).

Destacadamente, a interação entre área plantada de fumo e de algodão aparece positivamente relacionada às taxas de suicídio, o que dá indícios acerca da contribuição dos agrotóxicos à base de organofosforados nas mortes voluntárias, confirmando, entre outros estudos, os resultados obtidos por Park e Lester (2012). Conforme a Tabela 2, a proxy para organofosforados revelou que um aumento de $1 \%$ da área plantada de fumo e de algodão está ligado ao aumento de 0,015 ponto 
das ocorrências de suicídio por 100 mil habitantes, sendo que 0,004 são efeitos diretos e 0,011 , efeitos indiretos.

Já a taxa de fecundidade possui um efeito composto de $-3,728$ sobre a variável dependente. Desse total, 0,932 é efeito direto, enquanto 2,796 são efeitos indiretos. A associação entre o suicídio e a taxa de fecundidade também segue a linha dos argumentos de Durkheim, uma vez que um maior número de filhos tende a aumentar a coesão familiar, favorecendo, por sua vez, a solidificação das relações sociais que consequentemente atenuariam taxas de suicídio na sociedade (CHUANG; HUANG, 1996).

O tamanho da população urbana, por seu turno, segundo Durkheim (2005), atua como um indicador de isolamento social. Percebe-se que os municípios mais urbanizados tendem a apresentar maiores taxas de suicídio, de modo que o aumento de $1 \%$ no tamanho da população urbana está relacionado a um aumento de 0,042 ponto percentual na taxa de suicídio, sendo 0,010 de efeitos diretos e 0,031 de efeitos indiretos.

Apesar de divergirem dos resultados obtidos por Helbich et al. (2017), os quais avaliam que o risco de suicídio é menor em regiões mais urbanizadas, estão em conformidade com a teoria sociológica no sentido de que a forte incidência de mortes voluntárias nas cidades reflete o enfraquecimento da integração social no meio urbano (DURKHEIM, 2005). Outra explicação plausível se refere à concentração populacional nessas áreas e a dificuldade de acesso a bens e serviços públicos nos grandes centros urbanos em função da precariedade de infraestrutura (CECCON et al., 2014).

Verifica-se também que a interação espacial é importante, dada a significativa relevância da defasagem espacial do suicídio. Isso fica ainda mais claro diante de efeitos indiretos consideravelmente superiores aos efeitos diretos para a maioria das variáveis, já que, com exceção da taxa de fecundidade, nota-se que mudanças no nível de uma variável independente exercem impacto multiplicativo sobre todos os municípios na probabilidade de quase três vezes a magnitude do efeito direto sobre o próprio município. Esses resultados salientam o papel substancial desempenhado pelos efeitos dos multiplicadores espaciais, o que é consistente com a literatura do suicídio. Apesar da presença significativa da defasagem espacial não ser condição suficiente para inferir a presença de efeito-contágio, trata-se de resultado condizente com essa hipótese, apresentada teoricamente na revisão da literatura.

\section{Considerações Finais}

Em face do caráter epidêmico adquirido pelo suicídio nas últimas décadas, e de este ser reconhecido atualmente em todo o mundo como um problema de saúde pública (WORLD HEALTH ORGANIZATION, 2016), o presente estudo, com 
o objetivo de verificar as influências de aspectos socioeconômicos e demográficos na presença de interações espaciais, contribui para esse segmento da literatura, o qual carecia de aplicações ao nível municipal no Brasil. Apesar de a agregação utilizada não permitir inferências para os indivíduos, também logra avanços na medida em que utiliza uma metodologia adequada ao tratamento de dados censurados.

No que tange aos resultados, esses depõem em favor do caráter multifacetado das mortes voluntárias potencialmente associadas no espaço com aspectos macrossociais, sugerindo que estratégias de contenção e de prevenção devem ser pensadas em âmbito holístico e espacialmente inclusivo. De acordo com os achados deste estudo, o IDHM dimensão educação, o grau de formalização do trabalho, a taxa de fecundidade, a renda per capita, a área plantada de fumo e de algodão, bem como o tamanho da população urbana explicam significativamente os óbitos por suicídios nos municípios brasileiros, sendo as três primeiras inversamente relacionadas e as três últimas diretamente relacionadas com o fenômeno.

Nesse sentido, é desejável o fortalecimento das políticas destinadas a expandir a educação de qualidade, bem como adotar estratégias que visem à redução da informalidade no mercado de trabalho. Outro ponto a ser considerado é melhorar a fiscalização do uso de agrotóxicos e a restrição ao acesso a organofosforados, já que tais compostos estão associados às mortes voluntárias.

Tão importante quanto o emprego de ações multidimensionais é atentar para a distribuição territorial dessas ações, pois, conforme os resultados encontrados, políticas do tipo spatial blind voltadas inteiramente para as necessidades dos indivíduos podem não ser suficientes para surtir efeitos esperados sobre o suicídio. Isso ocorre em função da realidade do fenômeno nos municípios brasileiros não descartar a interação geográfica outrora postulada pelo pensamento durkheimiano.

Para estudos futuros, sugere-se o emprego de modelos que permitam captar a dinâmica temporal do suicídio ao longo do território brasileiro. Em que pesem as limitações metodológicas, a estratégia empírica adotada, distinta de estudos anteriores, mostrou-se relevante para o tratamento dos dados acometidos pela censura, o que, até então, constituía uma importante lacuna a ser preenchida na literatura. A especificação aqui proposta abre caminhos para trabalhos futuros ao mesmo tempo que os resultados obtidos podem ser utilizados pelos formuladores de políticas públicas como base para o desenho de políticas no âmbito municipal.

\section{Referências}

ABEL, E. L.; KRUGER, M. L. Educational attainment and suicide rates in the United States. Psychological Reports, v. 97, n. 1, p. 25-28, 2005. 
ABRUTYN, S.; MUELLER, A. S. Are suicidal behaviors contagious in adolescence? Using longitudinal data to examine suicide suggestion. American Sociological Review, v. 79, n. 2, p. 211-227, 2014.

ALMEIDA, L. N. et al. O suicídio no Brasil: Um desafio às Ciências Sociais. Revista Brasileira de Estudos Latino-Americanos, v. 5, n. 3, p. 510-591, 2015.

ALMEIDA, W. da S. de; SZWARCWALD, C. L. Adequação das informações de mortalidade e correção dos óbitos informados a partir da Pesquisa de Busca Ativa. Ciência \& Saúde Coletiva, v. 22, p. 3193-3203, 2017.

AMARAL, P. V.; ANSELIN, L. Finite sample properties of Moran's I test for spatial autocorrelation in TOBIT models. Papers in Regional Science, v. 93, n. 4, p. 773-781, 2014.

AMARAL, S. S. Suicídios no RN e sua relação com determinantes espaciais, urbanização, desenvolvimento e outros fatores socioeconômicos. In: ENCONTRO DA ASSOCIAÇÃO BRASILEIRA DE ESTUDOS REGIONAIS E URBANOS, 16., 2018, Caruaru. Anais [...]. Caruaru: SENAC, 2018.

AMEMIYA, T. Regression analysis when the dependent variable is truncated normal. Econometrica: Journal of the Econometric Society, v. 41, n. 6, p. 997-1016, 1973.

ANDRADE, C. L. T. de; SZWARCWALD, C. L. Desigualdades sócio-espaciais da adequação das informações de nascimentos e óbitos do Ministério da Saúde, Brasil, 2000-2002. Cadernos de Saúde Pública, v. 23, p. 1207-1216, 2007.

ANDRÉS, A. R. Income inequality, unemployment, and suicide: A panel data analysis of 15 European countries. Applied Economics, v. 37, n. 4, p. 439-451, 2005.

ANSELIN, L. Local Indicators of Spatial Association-LISA. Geographical Analysis, v. 27, n. 2, p. 93-115, 1995.

ANSELIN, L. Spatial econometrics. In: BALTAGI, B. H. (org.). A Companion to Theoretical Econometrics. Oxford: Blackwell Publishing, 2001. p. 310-330.

ANSELIN, L.; FLORAX, R. Small Sample Properties of Tests for Spatial Dependence in Regression Models: Some Further Results. In: ANSELIN, L.; FLORAX, R. (org.). New Directions in Spatial Econometrics. Heidelberg: Springer, 1995. p. 21-74.

ARAÚJO, V. de C.; GARCIA, R. Determinants and spatial dependence of innovation in Brazilian regions: Evidence from a Spatial Tobit Model. Nova Economia, v. 29, n. 2, p. 375-400, 2019.

BECKER, G. S.; POSNER, R. A. Suicide: An economic approach. Chicago: University of Chicago, 2004.

BENEDITO $\square$ SILVA, A. A.; NOGUEIRA PIRES, M. L.; CALIL, H. M. Seasonal variation of suicide in Brazil. Chronobiology International, v. 24, n. 4, p. 727-737, 2007. 
BIVAND, R. S. Exploratory Spatial Data Analysis. In: FISCHER, M. M.; GETIS, A. (org.). Handbook of Applied Spatial Analysis. Heidelberg: Springer, 2010. p. 219-254.

BOTEGA, N. J. Comportamento suicida: epidemiologia. Psicologia USP, v. 25, n. 3, p. 231236, 2014.

BOTEGA, N. J. Suicídio: saindo da sombra em direção a um Plano Nacional de Prevenção. Revista Brasileira de Psiquiatria, v. 29, n. 1, p. 7-8, 2007.

BRASIL. Ministério da Saúde. Datasus. O Sistema de Informações de Mortalidade. Brasília, DF, 2001. Disponível em: http://tabnet.datasus.gov.br/. Acesso em fevereiro de 2019.

CECCON, R. F. et al. Suicídio e trabalho em metrópoles brasileiras: um estudo ecológico. Ciência E Saúde Coletiva, v. 19, p. 2225-2234, 2014.

CHEN, J.; CHOI, Y. J.; SAWADA, Y. How is suicide different in Japan?. Japan and the World Economy, v. 21, n. 2, p. 140-150, 2009.

CHUANG, H.-L.; HUANG, W.-C. A reexamination of "sociological and economic theories of suicide: a comparison of the USA and Taiwan". Social Science $\mathcal{E}$ Medicine, v. 43, n. 3, p. 421-423, 1996.

CUTLER, D. M.; GLAESER, E. L.; NORBERG, K. E. Explaining the Rise in Youth Suicide. In: GRUBER, J. (org.). Risky Behavior Among Youths: An Economic Analysis. Chicago: University of Chicago Press, 2001. p. 219-270.

DALY, M. C.; WILSON, D. J.; JOHNSON, N. J. Relative status and well-being: Evidence from US suicide deaths. Review of Economics and Statistics, v. 95, n. 5, p. 1480-1500, 2013.

DE FRAGA, W. S.; MASSUQUETTI, A.; GODOY, M. R. Determinantes socioeconômicos do suicídio: um estudo para os municípios do Brasil e do Rio Grande do Sul. Revista Econômica, v. 18, n. 2, 2017.

DURKHEIM, E. Suicide: A study in sociology. Abingdon: Routledge, 2005.

FARIA, N. M. X.; FASSA, A. C. G.; MEUCCI, R. D. Association between pesticide exposure and suicide rates in Brazil. Neurotoxicology, v. 45, p. 355-362, 2014.

FLEMING, M. M. Techniques for Estimating Spatially Dependent Discrete Choice Models. In: ANSELIN, L.; FLORAX, R.; REY, S. J. (org.). Advances in Spatial Econometrics: Methodology, Tools and Applications. Heidelberg: Springer, 2004. p. 145-168.

FONTANELLA, C. A. et al. Widening rural-urban disparities in youth suicides, United States, 1996-2010. JAMA Pediatrics, v. 169, n. 5, p. 466-473, 2015.

FRIAS, P. G. de et al. Sistema de Informações sobre Mortalidade: estudo de caso em municípios com precariedade dos dados. Cadernos de Saúde Pública, v. 24, p. 2257-2266, 2008. 
FRIAS, P. G. de; SZWARCWALD, C. L.; LIRA, P. I. C. de. Avaliação dos sistemas de informações sobre nascidos vivos e óbitos no Brasil na década de 2000. Cadernos de Saúde Pública, v. 30, p. 2068-2280, 2014.

GOLGHER, A. B. Introdução à econometria espacial. Jundiaí: Paco Editorial, 2015.

GONÇALVES, L. R. C.; GONÇALVES, E.; OLIVEIRA JÚNIOR, L. B. de. Determinantes espaciais e socioeconômicos do suicídio no Brasil: uma abordagem regional. Nova Economia, v. 21, n. 2, p. 281-316, 2011.

GUNNELL, D. et al. Suicide and unemployment in young people: Analysis of trends in England and Wales, 1921-1995. The British Journal of Psychiatry, v. 175, n. 3, p. 263-270, 1999.

HAGHPARAST-BIDGOLI, H. et al. Socio-Demographic and Economic Factors Associated with Suicide Mortality in Iran, 2001-2010: Application of a Decomposition Model. International Journal for Equity in Health, v. 17, n. 1, p. 77, 2018.

HAMERMESH, D. S.; SOSS, N. M. An Economic Theory of Suicide. Journal of Political Economy, v. 82, n. 1, p. 83-98, 1974.

HELBICH, M. et al. Urban-Rural Inequalities in Suicide Mortality: A Comparison of Urbanicity Indicators. International Journal of Health Geographics, v. 16, n. 1, p. 39, 2017.

INSTITUTO BRASILEIRO DE GEOGRAFIA E ESTATÍSTICA (IBGE). SIDRA. Sistema IBGE de recuperação automática. Pesquisa Nacional por Amostra de Domicílios. 2010. Disponível em: <www.ibge.gov.br/sidra>. Acesso em fev. 2019.

JALLES, J. T.; ANDRESEN, M. A. The social and economic determinants of suicide in Canadian provinces. Health Economics Review, v. 5, n. 1, p. 1, 2015.

KYONNE, J. Impact of Social Service Expenditures on the Suicide Rate: The Cases of Asia Countries. Journal of Social Service Research, v. 45, n. 1, p. 12-15, 2019.

LEIGH, A.; JENCKS, C. Inequality and mortality: Long-run evidence from a panel of countries. Journal of Health Economics, v. 26, n. 1, p. 1-24, 2007.

LESAGE, J.; PACE, R. K. Introduction to Spatial Econometrics. Boca Raton, Florida: CRC Press, 2009.

LIN, J.-J.; LU, T.-H. Association between the accessibility to lethal methods and methodspecific suicide rates: An ecological study in Taiwan. The Journal of Clinical Psychiatry, v. 67, n. 7, p. 1074-1079, 2006.

LOUREIRO, P. R. A; DE MENDONÇA, M. J.C.; SACHSIDA, A. Os determinantes econômicos do suicídio: um estudo para o Brasil. Brasília, DF: Instituto de Pesquisa Econômica Aplicada, 2010.

LOUREIRO, P. R.A; MOREIRA, T. B.; SACHSIDA, A. Os efeitos da mídia sobre o suicídio: uma análise empírica para os estados brasileiros. Brasília, DF: Instituto de Pesquisa Econômica Aplicada, 2013. 
MACHADO, D. B.; RASELLA, D.; DOS SANTOS, D. N. Impact of income inequality and other social determinants on suicide rate in Brazil. PLoSOne, v. 10, n. 4, p. e0124934, 2015.

MELLO-SANTOS, C. de; BERTOLOTE, J. M.; WANG, Y.-P. Epidemiology of suicide in Brazil (1980-2000): Characterization of age and gender rates of suicide. Revista Brasileira de Psiquiatria, v. 27, n. 2, p. 131-134, 2005.

MINISTÉRIO DA SAÚDE. Norma Operacional de Assistência à Saúde/SUS-NOAS-SUS 01/2001. Brasília, 2001.

MINOIU, C.; ANDRES, A. R. The effect of public spending on suicide: Evidence from US state data. The Journal of Socio-Economics, v. 37, n. 1, p. 237-261, 2008.

MITRA, S.; SHROFF, S. Determinants of Suicide Rates in Developing Countries - an Econometric Investigation of the Indian Case. SSRN Papers, 2006.

ORAVECZ, A. et al. Constitutively Photomorphogenic is required for the UV-B response in Arabidopsis. The Plant Cell, v. 18, n. 8, p. 1975-1990, 2006.

PARK, B.C. B.; LESTER, D. Rural and urban suicide in South Korea. Psychological Reports, v. 111, n. 2, 2012.

PROGRAMA DAS NAÇÕES UNIDAS PARA O DESENVOLVIMENTO (PNUD). Atlas de Desenvolvimento Humano no Brasil. PNUD, 2010.

SANTOS, M. A. F. et al. Sofrimento no trabalho e imaginário organizacional: Ideação suicida de trabalhadora bancária. Psicologia E Sociedade, v. 23, n. 2, p. 359-368, 2011.

SCHNEIDER, B. et al. Impact of employment status and work-related factors on risk of completed suicide: A case-control psychological autopsy study. Psychiatry Research, v. 190, n. 2-3, p. 265-270, 2011.

SEARLES, V. B. et al. Suicides in urban and rural counties in the United States, 20062008. Crisis: The Journal of Crisis Intervention and Suicide Prevention, v. 35, n. 1, p. 18, 2014.

SETTI, V. M. G. et al. Políticas Públicas e prevenção do suicídio no Brasil. IIANDÉ: Ciências e Humanidades, v. 1, n. 1, p. 104-113, 2017.

SHIKIDA, C. D.; ARAUJO JR, A. F.; GAZZI, R. A. V. Teoria econômica do suicídio: estudo empírico para o Brasil. Análise Econômica, v. 25, n. 48, 2007.

SIQUEIRA, P. R. Mortalidade violenta no Brasil: Abordagens espaço-temporais. 2017. Dissertação (Mestrado em Economia) - Faculdade de Ciências Econômicas, Universidade Federal de Minas Gerais, Belo Horizonte, 2017.

SOUZA, E. R. de; MINAYO, M. C. de S.; MALAQUIAS, J. V. Suicídio de jovens nas principais capitais do Brasil. Cadernos de Saúde Pública, v. 18, n. 3, p. 673-683, 2002.

TOBIN, J. Estimation of Relationships for Limited Dependent Variables. Econometrica, v. 26, n. 1, p. 24-36, 1958. 
WORLD HEALTH ORGANIZATION. Preventing suicide: A global imperative. Geneva: World Health Organization, 2014.

WORLD HEALTH ORGANIZATION. World health statistics 2015. World Health Organization, 2015.

WORLD HEALTH ORGANIZATION. World health statistics 2016: Monitoring health for the SDGs sustainable development goals. Geneva: World Health Organization, 2016.

WILHELM, S.; DE MATOS, M. G. Estimating Spatial Probit Models in R. R Journal, v. 5, n. 1 , p. 130-143, 2013.

XU, X.; LEE, L. Maximum likelihood estimation of a spatial autoregressive Tobit model. Journal of Econometrics, v. 188, n. 1, p. 264-280, 2015.

YANG, B. The economy and suicide. American Journal of Economics and Sociology, v. 51, n. 1, p. 87-99, 1992.

\section{Autor correspondente:}

Samia Mercado Alvarenga

Recebido em: 11/06/2019.

E-mail: samia_alvarenga@hotmail.com

Aceito em: 11/03/2020.

\section{(cc) BY}

Este é um artigo de acesso aberto distribuído sob os termos da Creative Commons

Attribution CC-BY 4.0, que permite uso irrestrito, distribuição e reprodução em

qualquer meio, desde que o trabalho original seja devidamente citado. 